\title{
Choosing Wisely Canada's emergency medicine recommendations: Time for a revision
}

Amy H. Y. Cheng, MD, MBA*; Sam Campbell, MB, BCh, Dip PEC(SA) ${ }^{\dagger}$; Lucas B. Chartier, MDCM, $\mathrm{MPH}^{*}$; Shawn Dowling, MD; Tom Goddard, $\mathrm{MD}^{\dagger}$; Sophie Gosselin, MD ${ }^{\S}$; Brian R. Holroyd, MD, MBA"; Atul K. Kapur, MD, MScll; Kirk Magee, MD, MSc ${ }^{\dagger}$; Jill McEwen, MD**; Venkatesh Thiruganasambandamoorthy, MD, MScll; Suneel Upadhye, MD, MSc ${ }^{\dagger+}$; Brian H. Rowe, MD, MSc ${ }^{\ddagger \ddagger}$

\section{ABSTRACT}

Choosing Wisely Canada (CWC) is a national initiative designed to encourage patient-clinician discussions about the appropriate, evidence-based use of medical tests, procedures and treatments. The Canadian Association of Emergency Physicians' (CAEP) Choosing Wisely Canada (CWC) working group developed and released ten recommendations relevant to Emergency Medicine in June 2015 (items 1-5) and October 2016 (items 6-10). In November 2016, the CAEP CWC working group developed a process for updating the recommendations. This process involves: 1) Using GRADE to evaluate the quality of evidence, 2) reviewing relevant recommendations on an ad hoc basis as new evidence emerges, and 3) reviewing all recommendations every five years. While the full review of the CWC recommendations will be performed in 2020, a number of high-impact studies were published after our initial launch that prompted an ad hoc review of the relevant three of our ten recommendations prior to the full review in 2020. This paper describes the results of the CAEP CWC working group's ad hoc review of three of our ten recommendations in light of recent publications.

\section{RÉSUMÉ}

L'initiative nationale Choisir avec soin a été conçue pour favoriser les discussions entre patients et cliniciens sur l'utilisation appropriée et fondée sur des données probantes des examens médicaux, des interventions et des traitements. Le groupe de travail sur l'initiative, de l'Association canadienne des médecins d'urgence, a élaboré et diffusé dix recommandations relatives à la pratique de la médecine d'urgence, d'abord en juin 2015 (points 1-5), puis en octobre 2016 (points 6-10). En novembre 2016, le groupe de travail sur l'initiative s'est penché sur un processus de mise à jour des recommandations. Ce dernier comprend trois éléments : 1) l'application de l'instrument GRADE pour évaluer la qualité des données probantes; 2) une révision ponctuelle des recommandations pertinentes suivant la diffusion de nouvelles données; 3) un réexamen quinquennal de toutes les recommandations. La révision complète des recommandations présentées dans l'initiative est prévue en 2020; toutefois, un certain nombre d'études ayant une incidence importante ont déjà été publiées après le premier lancement des recommandations, ce qui a incité le groupe de travail à procéder à une révision ponctuelle de trois recommandations pertinentes sur les dix existantes, avant l'examen complet prévu en 2020. II sera donc question, dans l'article, des résultats de la révision ponctuelle de ces trois recommandations, réalisée à la lumière des récentes publications, par le groupe de travail sur l'initiative.

Keywords: Emergency medicine, evidence-based medicine

\section{INTRODUCTION}

Choosing Wisely Canada ${ }^{\circledR}$ (CWC) is a national initiative designed to encourage patient-clinician discussions about the appropriate, evidence-based use of medical tests, procedures, and treatments. Since its inception in 2014, the Canadian Association of Emergency Physicians' (CAEP) Choosing Wisely Canada working

From *Division of Emergency Medicine, Department of Medicine, University of Toronto, Toronto, ON; ${ }^{\dagger}$ Department of Emergency Medicine, Dalhousie University, Halifax, NS; ${ }^{\ddagger}$ Department of Emergency Medicine, University of Calgary, Calgary, AB; ${ }^{5}$ Department of Medicine, McGill University,

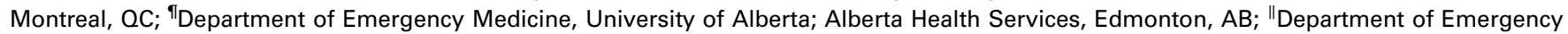
Medicine, University of Ottawa, Ottawa, ON; **Department of Emergency Medicine, University of British Columbia, Vancouver, BC; ${ }^{\dagger \dagger}$ Division of Emergency Medicine, Department of Medicine, McMaster University, Hamilton, ON; and the ${ }^{\ddagger \neq}$ Department of Emergency Medicine and School of Public Health, University of Alberta; Alberta Health Services, Edmonton, AB.

Correspondence to: Dr. Amy H Y Cheng, 30 Bond Street, 1-008 Shuter, Toronto, ON M5B1W8; Email: ChengAm@smh.ca

(C) Canadian Association of Emergency Physicians

CAEP | ACMU
CJEM 2019;21(6):717-720

DOI 10.1017/cem.2019.405
$2019 ; 21(6)$ 
group has developed and released 10 recommendations relevant to emergency medicine in June 2015 (items 1-5) and October 2016 (items 6-10). The detailed methods used to develop these recommendations were

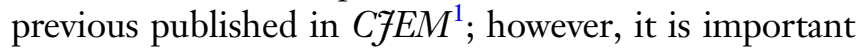
to understand that wide feedback from committee chairs and CAEP members was sought and that an evidence-based, consensus decision-making model was employed.

\section{METHODS}

Despite the use of the highest available evidence at the time of development, as new evidence has accumulated, the CAEP CWC working group recognized that a process for updating the recommendations was necessary. CWC also recognizes the importance of the regular review and update of recommendations by the specialist societies but recommends that the societies develop their own review method. In November 2016, the CAEP CWC working group developed a revision protocol to ensure that the CAEP CWC recommendations will remain up to date with evolving evidence. The process involves: 1) using GRADE ${ }^{2}$ to evaluate the quality of evidence; 2) reviewing relevant recommendations on an ad hoc basis as new evidence emerges; and 3) reviewing all recommendations every five years. Each working group member is assigned to 1 of the 10 recommendations and is responsible for monitoring the evidence and alerting the working group when a high quality paper is published. The CWC working group will review the evidence using GRADE. Expert opinion on specific recommendations is encouraged from those outside the CAEP CWC working group, as needed. The final decision to make revisions to any recommendation(s) rests with members of the working group using a consensusbased process.

Since the release of the CWC emergency medicine recommendations in 2015, a few high-impact articles and guidelines were published that were potentially relevant to three of our recommendations. The CAEP CWC working group felt that a more detailed review of these articles was needed prior to the full review scheduled for 2020. Therefore, we reviewed the articles and the three relevant CWC guidelines, sought the opinion of external experts, and revised the justifications of the recommendations based on our review.

\section{RESULTS}

For the recommendation related to computed tomography (CT) head scans for minor head injuries, we reviewed the external validation study of the three clinical decision rules in children by Babl et al. ${ }^{3}$ For the recommendation related to the use of antibiotics for sore throats, we reviewed the most recent and Canadianrelevant antimicrobial treatment guidelines and worked with other Canadian specialist societies including the Association of Medical Microbiology and Infectious Disease Canada and CWC to ensure that our recommendation was consistent with those from other specialties (pediatrics). ${ }^{4}$ Finally, we also reviewed the two randomized control trials and a systematic review related to the use of antibiotics, in addition to incision and drainage, for the treatment for simple abscesses. ${ }^{5-7}$

\section{DISCUSSION}

\section{CT head scans for minor head injuries}

Recommendation (unchanged): Don't order CT head scans in adults and children who have suffered minor head injuries (unless positive for a validated head injury clinical decision rule)

fustification (revised): Head injuries in children and adults are common presentations to the emergency department. Minor head injury is characterized by: Glasgow Coma Scale (GCS) 1315, an event that is associated with either witnessed loss of consciousness, definite amnesia, or witnessed disorientation. Most adults and children with minor head injuries do not suffer from serious brain injuries that require hospitalization or surgery. CT head scans performed on patients who lack high-risk features can expose patients to unnecessary ionizing radiation that has the potential to increase patients' lifetime risk of cancer. They also increase length of stay and increase the detection of false-positives (incidental, non-clinically relevant findings). There is strong evidence that physicians should not order CT head scans for patients with minor head injury unless validated clinical decision rules are used to make imaging decisions (i.e., Canadian CT head rule for adults, and Canadian Assessment of Tomography for Childhood Head Injury (CATCH) and/or PECARN rules for children). However, CATCH has been shown to be less sensitive than PECARN at detecting any brain injury on $C T$. While we recommend the use of clinical decision rules (CDRs) for head injuries, these rules are meant to assist and not replace, clinical judgment. 


\section{Literature review findings}

Our goal in this recommendation was to reduce the use of advanced imaging in the absence of evidence-based indicators in minor head injuries in an attempt to: reduce patient ED length of stay, reduce needless radiation exposure, and encourage resource stewardship. We had recommended using the Canadian CT head rule for adults and CATCH and/or PECARN for children. Since the release of our recommendation, a large $(n=$ $20,137)$ external validation of the three clinical decision rules (PECARN, CATCH, and Children's Head injury ALgorithm for the prediction of Important Clinical Events [CHALICE]) in children showed that PECARN is the most sensitive $(100 \%$ sensitivity in patients aged less than 2 years; $99 \%$ in those aged more than 2 years), followed by CATCH and CHALICE. ${ }^{3}$ Furthermore, the authors of the original CATCH rule had also recently showed that the original CATCH rule was validated poorly in Canada $(91.3 \%$ sensitive for neurosurgical intervention, and $97.5 \%$ for predicting brain injury). ${ }^{8}$

We discussed this evolving evidence and concluded that a direct comparison of the three CDRs, PECARN, CATCH, and CHALICE, is not valid. The PECARN tool was designed to identify children who should not get a CT scan, but CATCH was designed to identify children who should receive a CT scan. Furthermore, CATCH, when applied as published, is more restrictive and was only applicable in $25 \%$ of the study population (compared with 75\% PECARN and 99\% CHALICE). ${ }^{3}$ Therefore, as a direct comparison of both rules was deemed to be invalid, we recommend that clinicians use either one of these rules (CATCH or PECARN) while considering the use of CT head scans in the pediatric population, with the understanding of the limitations of each. CATCH 2, although recently found to be more sensitive than the original CATCH, cannot be recommended as it has not been externally validated. ${ }^{8}$

\section{Antibiotics for sore throats}

Recommendation (unchanged): Don't use antibiotics in adults and children with uncomplicated sore throats

Fustification (revised): Adults and children frequently present to the emergency department with sore throats (pharyngitis). The vast majority of cases of pharyngitis are caused by self-limiting viral infections that do not respond to antibiotics. The benefit of antibiotics for the approximately $10 \%$ of cases in adults ( $25 \%$ in children), caused by bacteria (principally Group A Streptococcus [GAS]) is modest at best, although they are associated with fewer complications and a slightly shorter course of illness. Inappropriate administration of antibiotics can expose patients to unnecessary risks (i.e., allergies, rash, and diarrhea) and increase overall antibiotic resistance in the community. Evidence suggests that antibiotics should only be used in patients with intermediate and high clinical prediction scores for GAS (CENTOR or FeverPAIN score) AND confirmatory testing (throat cultures or rapid testing) that is positive for GAS.

\section{Literature review findings}

Our goal in this recommendation was to reduce the use of antibiotics in an attempt to: reduce adverse effects experienced by patients and encourage antibiotic stewardship by physicians. Acute pharyngitis is often caused by viruses and self-limiting. We had originally recommended using antibiotics to treat streptococcus A pharyngitis only in patients with high clinical prediction scores for GAS (CENTOR ${ }^{9}$ or FeverPAIN score $^{10}$ ) or intermediate clinical scores AND positive throat cultures.

There is now evidence to suggest that patients with intermediate and high clinical prediction scores for GAS require confirmatory testing (rapid strep OR throat cultures) before prescribing antibiotics. ${ }^{4} \mathrm{We}$ believe this approach will further decrease the use of unnecessary antibiotics and have thus revised our recommendation accordingly.

\section{Antibiotics for treating simple abscesses after incision and drainage}

Recommendation (unchanged): Don't prescribe antibiotics after incision and drainage of uncomplicated skin abscesses unless extensive cellulitis exists

fustification (revised): Abscesses are walled off collections of pus in soft tissue, with Staphylococcus aureus (both sensitive and resistant to methicillin) being the microbe most frequently involved. Most uncomplicated abscesses should undergo incision in an acute care setting such as the emergency department, using local anesthesia or procedural sedation, with complete drainage and appropriate follow-up. Antibiotics may be considered when patients are immunocompromised, systemically ill, or exhibit extensive surrounding cellulitis or lymphangitis. In 
populations with a high [methicillin-resistant S. aureus] $M R S A$ prevalence, there is some evidence to suggest that antibiotics in addition to incision and drainage of uncomplicated abscesses may confer some benefit. However, we encourage physicians to discuss the use of antibiotics in uncomplicated abscesses with patients as the benefits conferred by antibiotics may not outweigh the risks associated with their use (i.e. nausea, diarrhea, and allergic reactions).

\section{Literature review findings}

Our goal in this recommendation was to reduce the use of antibiotics in an attempt to reduce adverse effects experienced by patients and encourage antibiotic stewardship by physicians. We initially recommended that incision and drainage (I\&D) with adequate follow-up be used to treat uncomplicated skin abscesses. We recommended that antibiotics be used in addition to I\&D when treating complicated cases only (i.e., immunocompromised, systemically ill, or exhibit extensive surrounding cellulitis or lymphangitis).

Recently, two randomized controlled trials and a systematic review demonstrated that the use of trimethoprim-sulfamethoxazole (TMP-SMX), in addition to I\&D, may confer some benefits compared with I\&D alone. ${ }^{5-7}$ Adding antibiotics results in a modest reduction in pain and treatment failure but also results in a significant increase in adverse effects such as nausea and diarrhea. Furthermore, the generalizability of the study results may be limited, as the populations in both studies had a rate of MRSA exceeding $40 \%$ that is much higher than in many areas of Canada. Therefore, we recommend shared decision-making: that physicians discuss the use of antibiotics in uncomplicated abscesses with patients as the benefits conferred by antibiotics may not outweigh the risks associated with their use (i.e., nausea, diarrhea, and allergic reactions).

\section{CONCLUSION}

Since the release of the CWC emergency medicine recommendations in 2015, a few high-impact articles were published that were relevant to the topics of 3 of our 10 recommendations. The CAEP CWC working group felt that a more detailed review of these articles was needed prior to the full review of all recommendations scheduled for 2020. After a thorough review of the new literature and discussions with external experts, the CAEP CWC working group revised the justifications for the three recommendations while retaining the original wording of the three recommendations. As per the review process, a full review of the 10 CAEP CWC recommendations will take place in 2020, and we welcome input from CAEP members on the review and revisions.

Supplementary material: The supplementary material for this article can be found at https://doi.org/10.1017/cem.2019.405.

Competing interests: None of the members of the CAEP CWC working group have any known conflicts of interest to declare. No direct funding was received for this work. Dr. Rowe was supported as Tier I Canada Research Chair in Evidence-based Emergency Medicine by the Canadian Institutes of Health Research (CIHR) through the Government of Canada (Ottawa, ON). The funders take no responsibility for the content and opinions expressed here.

\section{REFERENCES}

1. Cheng A, Campbell S, Chartier L, et al. Choosing Wisely Canada (C): five tests, procedures and treatments to question in emergency medicine. CFEM 2017;19 S2:S9-17.

2. Guyatt GH, Oxman AD, Vist GE, et al. GRADE: an emerging consensus on rating quality of evidence and strength of recommendations. BM7 2008;336(7650):924-6.

3. Babl FE, Borland ML, Phillips N, et al. Accuracy of PECARN, CATCH, and CHALICE head injury decision rules in children: a prospective cohort study. Lancet 2017;389(10087):2393-402.

4. Institut national d'excellence en sante et en services sociaux (INESS). Pharyngite-Amygdalite Chez L'enfant Et L'adulte. Conseil du Medicament, Quebec; 2016.

5. Talan DA, Mower WR, Krishnadasan A, et al. Trimethoprimsulfamethoxazole versus placebo for uncomplicated skin abscess. N Engl 7 Med 2016;374(9):823-32.

6. Vermandere M, Aertgeerts B, Agoritsas T, et al. Antibiotics after incision and drainage for uncomplicated skin abscesses: a clinical practice guideline. BM7 2018;360:k243.

7. Daum RS, Miller LG, Immergluck L, et al. A placebocontrolled trial of antibiotics for smaller skin abscesses. $N$ Engl 7 Med 2017;376(26):2545-55.

8. Osmond MH, Klassen TP, Wells GA, et al. Validation and refinement of a clinical decision rule for the use of computed tomography in children with minor head injury in the emergency department. CMA7 2018;190:E816-22.

9. Centor RM, Witherspoon JM, Dalton HP, Brody CE, Link K. The diagnosis of strep throat in adults in the emergency room. Med Decis Making 1981;1(3):239-46.

10. Little P, Moore M, Hobbs FD, et al. PRImary care Streptococcal Management (PRISM) study: identifying clinical variables associated with Lancefield group A $\beta$-haemolytic streptococci and Lancefield non-Group A streptococcal throat infections from two cohorts of patients presenting with an acute sore throat. BM7 Open 2013;3(10):e003943. 